\title{
The UTfit collaboration average of $D$ meson mixing data: spring 2012
}

\section{$\mathrm{UT}_{\text {fit }}$}

\author{
A.J. Bevan, ${ }^{a}$ M. Bona, ${ }^{a}$ M. Ciuchini, ${ }^{b}$ D. Derkach, ${ }^{c}$ E. Franco, ${ }^{d}$ V. Lubicz,,${ }^{b} e$
}

G. Martinelli, ${ }^{d, f}$ F. Parodi, ${ }^{g}$ M. Pierini, ${ }^{c}$ C. Schiavi, ${ }^{g}$ L. Silvestrini, ${ }^{d}$ V. Sordini, ${ }^{h}$

A. Stocchi, ${ }^{i}$ C. Tarantino ${ }^{b, e}$ and V. Vagnoni ${ }^{j}$

${ }^{a}$ Queen Mary, University of London, Mile End Road, London E1 4NS, U.K.

${ }^{b}$ INFN, Sezione di Roma Tre, Via della Vasca Navale 84, I-00146 Roma, Italy

${ }^{c}$ CERN, CH-1211 Geneva 23, Switzerland

${ }^{d}$ INFN, Sezione di Roma, Piazzale A. Moro 2, I-00185 Roma, Italy

e Dipartimento di Fisica, Università di Roma Tre, Via della Vasca Navale 84, I-00146 Roma, Italy

${ }^{f}$ SISSA-ISAS, Via Bonomea 265, I-34136 Trieste, Italy

${ }^{g}$ Dipartimento di Fisica, Università di Genova and INFN,

Via Dodecaneso 33, I-16146 Genova, Italy

${ }^{h}$ IPNL-IN2P3, 4 Rue Enrico Fermi, F-69622 Villeurbanne Cedex, France

${ }^{i}$ Laboratoire de l'Accélérateur Linéaire, IN2P3-CNRS et Université de Paris-Sud, BP 34, F-91898 Orsay Cedex, France

${ }^{j}$ INFN, Sezione di Bologna, Via Irnerio 46, I-40126 Bologna, Italy

E-mail: A.J.Bevan@qmul.ac.uk, Marcella.Bona@cern.ch, ciuchini@roma3.infn.it, Derkach@lal.in2p3.fr,

Enrico.Franco@roma1.infn.it, Lubicz@fis.uniroma3.it, Guido.Martinelli@sissa.it, Fabrizio.Parodi@cern.ch, Maurizio.Pierini@cern.ch, Carlo.Schiavi@ge.infn.it, Luca.Silvestrini@roma1.infn.it, Viola.Sordini@cern.ch, Achille.Stocchi@lal.in2p3.fr, tarantino@fis.uniroma3.it, Vincenzo.Vagnoni@bo.infn.it

AbStract: We derive constraints on the parameters $M_{12}, \Gamma_{12}$ and $\Phi_{12}$ that describe $D$ meson mixing using all available data, allowing for $\mathrm{CP}$ violation. We also provide posterior distributions and predictions for observable parameters appearing in $D$ physics.

KeYwords: Quark Masses and SM Parameters, Heavy Quark Physics, CP violation ARXIV EPRINT: 1206.6245

\footnotetext{
${ }^{1}$ Collaboration homepage: http://www.utfit.org/.
} 
Meson-antimeson mixing in the neutral $D$ system has been established only in 2007 [13]. Early combinations of available data allowed to put stringent constraints on New Physics (NP) contributions, although the possibility of non-standard CP violation remained open [4-8]. More recently, CP violation in the $D$ system received considerable attention after the measurement at hadron colliders of large direct $\mathrm{CP}$ violation in $D \rightarrow \pi \pi$ and $D \rightarrow K K$ decays $[9,10]$, which may signal the presence of NP [11-16]. It then becomes crucial to extract updated information on the mixing amplitude in order both to disentangle more precisely indirect and direct $\mathrm{CP}$ violation in $D \rightarrow \pi \pi$ and $D \rightarrow K K$, and to obtain up-to-date constraints on NP in $\Delta C=2$ transitions that can be used to constrain NP contributions to $\Delta C=1$ processes in any given model.

In this letter, we perform a fit to the experimental data in table 1 following the statistical method described in ref. [39]. A number of assumptions can be made in order to combine the measurements in table 1. First of all, let us assume that Cabibbo allowed (CA) and doubly Cabibbo suppressed (DCS) decays are purely tree-level SM processes, so that there is no direct $\mathrm{CP}$ violation (for the moment, we do not specify any phase convention). Furthermore, we neglect the weak phase difference between these channels, which is of $\mathcal{O}\left(10^{-3}\right)$. Then, for these decay channels, one has

$$
\lambda_{f}=\frac{q}{p} \frac{\bar{A}_{f}}{A_{f}}=\left|\frac{q}{p}\right| R_{f} e^{i\left(\phi+\delta_{f}\right)}, \quad \lambda_{\bar{f}}=\frac{q}{p} \frac{\bar{A}_{\bar{f}}}{A_{\bar{f}}}=\left|\frac{q}{p}\right| R_{f}^{-1} e^{i\left(\phi-\delta_{f}\right)},
$$

where $A_{f}=A(D \rightarrow f)$ corresponds to a Cabibbo allowed (or doubly Cabibbo suppressed) decay, $\bar{A}_{f}=A(\bar{D} \rightarrow f), R_{f}=\left|\bar{A}_{f} / A_{f}\right|, \delta_{f}$ is the strong phase and $\phi$ is the relative weak phase between mixing and decay. One can then write the following equations [4, 40-43], with $\left|D_{L, S}\right\rangle=p\left|D^{0}\right\rangle \pm q\left|\bar{D}^{0}\right\rangle$ and $|p|^{2}+|q|^{2}=1$ :

$$
\begin{array}{rlrl}
\delta & =\frac{1-|q / p|^{2}}{1+|q / p|^{2}}, \quad \arg \left(\Gamma_{12} q / p\right)=\arg (y+i \delta x), & A_{M}=\frac{|q / p|^{4}-1}{|q / p|^{4}+1}, \quad R_{M}=\frac{x^{2}+y^{2}}{2}, \\
\left(\begin{array}{c}
x_{f}^{\prime} \\
y_{f}^{\prime}
\end{array}\right) & =\left(\begin{array}{cc}
\cos \delta_{f} & \sin \delta_{f} \\
-\sin \delta_{f} & \cos \delta_{f}
\end{array}\right)\left(\begin{array}{c}
x \\
y
\end{array}\right), & \left(x_{ \pm}^{\prime}\right)_{f}=\left|\frac{q}{p}\right|^{ \pm 1}\left(x_{f}^{\prime} \cos \phi \pm y_{f}^{\prime} \sin \phi\right), \\
\left(y_{ \pm}^{\prime}\right)_{f} & =\left|\frac{q}{p}\right|^{ \pm 1}\left(y_{f}^{\prime} \cos \phi \mp x_{f}^{\prime} \sin \phi\right), \\
y_{\mathrm{CP}} & =\left(\left|\frac{q}{p}\right|+\left|\frac{p}{q}\right|\right) \frac{y}{2} \cos \phi-\left(\left|\frac{q}{p}\right|-\left|\frac{p}{q}\right|\right) \frac{x}{2} \sin \phi, \\
A_{\Gamma} & =\left(\left|\frac{q}{p}\right|-\left|\frac{p}{q}\right|\right) \frac{y}{2} \cos \phi-\left(\left|\frac{q}{p}\right|+\left|\frac{p}{q}\right|\right) \frac{x}{2} \sin \phi,
\end{array}
$$

valid for Cabibbo allowed and doubly Cabibbo suppressed final states.

Let us now choose for convenience a specific phase convention: $\mathrm{CP}|D\rangle=|\bar{D}\rangle$ and the standard CKM phase convention, so that CA and DCS decay amplitudes have vanishing weak phase and $\phi=\arg (q / p)$.

For singly Cabibbo suppressed decays $D^{0} \rightarrow K^{+} K^{-}$and $D^{0} \rightarrow \pi^{+} \pi^{-}$we allow for direct $\mathrm{CP}$ violation to be present. Direct $\mathrm{CP}$ violation requires a subdominant amplitude: $A_{f}=A_{\text {tree }}\left(1+r_{f} e^{i\left(\phi_{f}+\delta_{f}\right)}\right), \bar{A}_{f}=A_{\text {tree }}\left(1+r_{f} e^{i\left(-\phi_{f}+\delta_{f}\right)}\right)$. Present experimental results 


\begin{tabular}{|c|c|c|c|c|c|c|c|}
\hline Observable & Value & \multicolumn{5}{|c|}{ Correlation Coeff. } & Reference \\
\hline$y_{C P}$ & $(0.866 \pm 0.155) \%$ & & & & & & {$[2,17-25]$} \\
\hline$A_{\Gamma}$ & $(0.022 \pm 0.161) \%$ & & & & & & {$[2,20,23-26]$} \\
\hline$x$ & $(0.811 \pm 0.334) \%$ & 1 & -0.007 & $-0.255 \alpha$ & 0.216 & & {$[3]$} \\
\hline$y$ & $(0.309 \pm 0.281) \%$ & -0.007 & 1 & $-0.019 \alpha$ & -0.280 & & {$[3]$} \\
\hline$|q / p|$ & $(0.95 \pm 0.22 \pm 0.10) \%$ & $-0.255 \alpha$ & $-0.019 \alpha$ & 1 & $-0.128 \alpha$ & & {$[3]$} \\
\hline$\phi$ & $(-0.035 \pm 0.19 \pm 0.09)$ & 0.216 & -0.280 & $-0.128 \alpha$ & 1 & & {$[3]$} \\
\hline$x$ & $(0.16 \pm 0.23 \pm 0.12 \pm 0.08) \%$ & 1 & 0.0615 & & & & {$[27]$} \\
\hline$y$ & $(0.57 \pm 0.20 \pm 0.13 \pm 0.07) \%$ & 0.0615 & 1 & & & & {$[27]$} \\
\hline$R_{M}$ & $(0.0130 \pm 0.0269) \%$ & & & & & & {$[28-32]$} \\
\hline$\left(x_{+}^{\prime}\right)_{K \pi \pi^{0}}$ & $(2.48 \pm 0.59 \pm 0.39) \%$ & 1 & -0.69 & & & & {$[33]$} \\
\hline$\left(y_{+}^{\prime}\right)_{K \pi \pi^{0}}$ & $(-0.07 \pm 0.65 \pm 0.50) \%$ & -0.69 & 1 & & & & {$[33]$} \\
\hline$\left(x_{-}^{\prime}\right)_{K \pi \pi^{0}}$ & $(3.50 \pm 0.78 \pm 0.65) \%$ & 1 & -0.66 & & & & {$[33]$} \\
\hline$\left(y_{-}^{\prime}\right)_{K \pi \pi^{0}}$ & $(-0.82 \pm 0.68 \pm 0.41) \%$ & -0.66 & 1 & & & & {$[33]$} \\
\hline$x^{2}$ & $(0.1549 \pm 0.2223) \%$ & 1 & -0.6217 & -0.00224 & 0.3698 & 0.01567 & {$[34]$} \\
\hline$y$ & $(2.997 \pm 2.293) \%$ & -0.6217 & 1 & 0.00414 & -0.5756 & -0.0243 & {$[34]$} \\
\hline$R_{D}$ & $(0.4118 \pm 0.0948) \%$ & -0.00224 & 0.00414 & 1 & 0.0035 & 0.00978 & {$[34]$} \\
\hline $2 \sqrt{R_{D}} \cos \delta_{K \pi}$ & $(12.64 \pm 2.86) \%$ & 0.3698 & -0.5756 & 0.0035 & 1 & 0.0471 & {$[34]$} \\
\hline $2 \sqrt{R_{D}} \sin \delta_{K \pi}$ & $(-0.5242 \pm 6.426) \%$ & 0.01567 & -0.0243 & 0.00978 & 0.0471 & 1 & {$[34]$} \\
\hline$R_{D}$ & $(0.3030 \pm 0.0189) \%$ & 1 & 0.77 & -0.87 & & & {$[1]$} \\
\hline$\left(x_{+}^{\prime}\right)_{K \pi}^{2}$ & $(-0.024 \pm 0.052) \%$ & 0.77 & 1 & -0.94 & & & {$[1]$} \\
\hline$\left(y_{+}^{\prime}\right)_{K \pi}$ & $(0.98 \pm 0.78) \%$ & -0.87 & -0.94 & 1 & & & {$[1]$} \\
\hline$A_{D}$ & $(-2.1 \pm 5.4) \%$ & 1 & 0.77 & -0.87 & & & {$[1]$} \\
\hline$\left(x_{-}^{\prime}\right)_{K \pi}^{2}$ & $(-0.020 \pm 0.050) \%$ & 0.77 & 1 & -0.94 & & & {$[1]$} \\
\hline$\left(y_{-}^{\prime}\right)_{K \pi}$ & $(0.96 \pm 0.75) \%$ & -0.87 & -0.94 & 1 & & & {$[1]$} \\
\hline$R_{D}$ & $(0.364 \pm 0.018) \%$ & 1 & 0.655 & -0.834 & & & {$[35]$} \\
\hline$\left(x_{+}^{\prime}\right)_{K \pi}^{2}$ & $(0.032 \pm 0.037) \%$ & 0.655 & 1 & -0.909 & & & {$[35]$} \\
\hline$\left(y_{+}^{\prime}\right)_{K \pi}$ & $(-0.12 \pm 0.58) \%$ & -0.834 & -0.909 & 1 & & & {$[35]$} \\
\hline$A_{D}$ & $(2.3 \pm 4.7) \%$ & 1 & 0.655 & -0.834 & & & {$[35]$} \\
\hline$\left(x_{-}^{\prime}\right)_{K \pi}^{2}$ & $(0.006 \pm 0.034) \%$ & 0.655 & 1 & -0.909 & & & {$[35]$} \\
\hline$\left(y_{-}^{\prime}\right)_{K \pi}$ & $(0.20 \pm 0.54) \%$ & -0.834 & -0.909 & 1 & & & {$[35]$} \\
\hline CP asymmetry & Value & & & $\Delta\langle t\rangle / \tau_{D^{0}}$ & & & Reference \\
\hline$A_{\mathrm{CP}}\left(D^{0} \rightarrow K^{+} K^{-}\right)$ & $(-0.24 \pm 0.24) \%$ & & & & & & {$[36,37]$} \\
\hline$A_{\mathrm{CP}}\left(D^{0} \rightarrow \pi^{+} \pi^{-}\right)$ & $(0.11 \pm 0.39) \%$ & & & & & & {$[36,37]$} \\
\hline$\Delta A_{\mathrm{CP}}$ & $(-0.82 \pm 0.21 \pm 0.11) \%$ & & $9.83 \pm 0.2$ & $22 \pm 0.19) \%$ & & & {$[9]$} \\
\hline$\Delta A_{\mathrm{CP}}$ & $(-0.62 \pm 0.21 \pm 0.10) \%$ & & $(26 \pm$ & $\pm 1) \%$ & & & {$[10]$} \\
\hline
\end{tabular}

Table 1. Experimental data used in the analysis of $D$ mixing, from ref. [38]. ${ }^{1} \alpha=(1+|q / p|)^{2} / 2$ and $\Delta A_{\mathrm{CP}}=A_{\mathrm{CP}}\left(D^{0} \rightarrow K^{+} K^{-}\right)-A_{\mathrm{CP}}\left(D^{0} \rightarrow \pi^{+} \pi^{-}\right)$. Asymmetric errors have been symmetrized. We do not use measurements that do not allow for CP violation in mixing, except for ref. [27] (as shown in ref. [3], the results for $x$ and $y$ from the Dalitz analysis of $D \rightarrow K_{s} \pi \pi$ are not sensitive to the assumptions about $\mathrm{CP}$ violation in mixing). 
imply $r_{f} \sin \delta_{f} \sin \phi_{f} \sim 6 \times 10^{-3}$. These amplitudes also contribute to $\Gamma_{12}$, possibly leading to $\arg \left(\Gamma_{12}\right) \leq \mathcal{O}\left(10^{-3}\right)$. Given the present experimental accuracy, one can then assume $\Gamma_{12}$ to be real, leading to the relation

$$
\phi=\arg (y+i \delta x) .
$$

We assume flat priors for $x=\Delta m_{D} / \Gamma_{D}, y=\Delta \Gamma_{D} /\left(2 \Gamma_{D}\right)$ and $|q / p|$. We can then express all mixing-related observables in terms of $x, y$ and $|q / p|$ using the formulæ above. Furthermore,

$$
R_{D}=\frac{\Gamma\left(D^{0} \rightarrow K^{+} \pi^{-}\right)+\Gamma\left(\bar{D}^{0} \rightarrow K^{-} \pi^{+}\right)}{\Gamma\left(D^{0} \rightarrow K^{-} \pi^{+}\right)+\Gamma\left(\bar{D}^{0} \rightarrow K^{+} \pi^{-}\right)}, \quad A_{D}=\frac{\Gamma\left(D^{0} \rightarrow K^{+} \pi^{-}\right)-\Gamma\left(\bar{D}^{0} \rightarrow K^{-} \pi^{+}\right)}{\Gamma\left(D^{0} \rightarrow K^{+} \pi^{-}\right)+\Gamma\left(\bar{D}^{0} \rightarrow K^{-} \pi^{+}\right)},
$$

with $A_{\mathrm{D}}$ forced to vanish in the fit. In addition, for the CP asymmetries we have

$$
A_{\mathrm{CP}}(f)=\frac{\Gamma\left(D^{0} \rightarrow f\right)-\Gamma\left(\bar{D}^{0} \rightarrow \bar{f}\right)}{\Gamma\left(D^{0} \rightarrow f\right)+\Gamma\left(\bar{D}^{0} \rightarrow \bar{f}\right)} \approx a_{\mathrm{CP}}^{\mathrm{dir}}(f)-A_{\Gamma} \int_{0}^{\infty} d t \frac{t}{\tau_{D^{0}}} D_{f}(t)=a_{\mathrm{CP}}^{\operatorname{dir}}(f)-\frac{\langle t\rangle_{f}}{\tau_{D^{0}}} A_{\Gamma},
$$

where $D_{f}(t)$ is the observed distribution of proper decay time and $\tau_{D^{0}}$ is the lifetime of the neutral $D$ mesons.

For the purpose of constraining NP, it is useful to express the fit results in terms of the $\Delta C=2$ effective Hamiltonian matrix elements $M_{12}$ and $\Gamma_{12}$ :

$$
\begin{aligned}
\left|M_{12}\right| & =\frac{1}{\tau_{D}} \sqrt{\frac{x^{2}+\delta^{2} y^{2}}{4\left(1-\delta^{2}\right)}}, \quad\left|\Gamma_{12}\right|=\frac{1}{\tau_{D}} \sqrt{\frac{y^{2}+\delta^{2} x^{2}}{1-\delta^{2}}}, \\
\sin \Phi_{12} & =\frac{\left|\Gamma_{12}\right|^{2}+4\left|M_{12}\right|^{2}-\left(x^{2}+y^{2}\right)|q / p|^{2} / \tau_{D}^{2}}{4\left|M_{12} \Gamma_{12}\right|},
\end{aligned}
$$

with $\Phi_{12}=\arg \Gamma_{12} / M_{12}$. Consistently with the assumptions above, $\Gamma_{12}$ can be taken real with negligible NP contributions, and a nonvanishing $\Phi_{12}$ can be interpreted as a signal of new sources of CP violation in $M_{12}$. For the sake of completeness, we report here also the formulæ to compute the observables $x, y$ and $\delta$ from $M_{12}$ and $\Gamma_{12}$ :

$$
\begin{aligned}
\sqrt{2} \Delta m & =\operatorname{sign}\left(\cos \Phi_{12}\right) \sqrt{4\left|M_{12}\right|^{2}-\left|\Gamma_{12}\right|^{2}+\sqrt{\left(4\left|M_{12}\right|^{2}+\left|\Gamma_{12}\right|^{2}\right)^{2}-16\left|M_{12}\right|^{2}\left|\Gamma_{12}\right|^{2} \sin ^{2} \Phi_{12}}} \\
\sqrt{2} \Delta \Gamma & =2 \sqrt{\left|\Gamma_{12}\right|^{2}-4\left|M_{12}\right|^{2}+\sqrt{\left(4\left|M_{12}\right|^{2}+\left|\Gamma_{12}\right|^{2}\right)^{2}-16\left|M_{12}\right|^{2}\left|\Gamma_{12}\right|^{2} \sin ^{2} \Phi_{12}}} \\
\delta & =\frac{2\left|M_{12}\right|\left|\Gamma_{12}\right| \sin \Phi_{12}}{(\Delta m)^{2}+\left|\Gamma_{12}\right|^{2}}
\end{aligned}
$$

in agreement with [42] up to a factor of $\sqrt{2}$.

The results of the fit are reported in table 2. The corresponding p.d.f are shown in figures 1 and 2. Some two-dimensional correlations are displayed in figure 3.

A direct comparison with the HFAG results $[38]^{1}$ is not straightforward, as our fit does not fall into any of the HFAG categories (no CPV, no direct CPV, direct CPV), since we allow for direct $\mathrm{CP}$ violation only in singly Cabibbo suppressed decays. However, our fit results should be close to the "no direct CPV" HFAG fit. Indeed, we find

\footnotetext{
${ }^{1}$ And online updates at http://www.slac.stanford.edu/xorg/hfag/.
} 


\begin{tabular}{|ccc|}
\hline parameter & result @ 68\% prob. & $95 \%$ prob. range \\
\hline$\left|M_{12}\right|[1 / \mathrm{ps}]$ & $(6.9 \pm 2.4) \cdot 10^{-3}$ & {$[2.1,11.5] \cdot 10^{-3}$} \\
$\left|\Gamma_{12}\right|[1 / \mathrm{ps}]$ & $(17.2 \pm 2.5) \cdot 10^{-3}$ & {$[12.3,22.4] \cdot 10^{-3}$} \\
$\Phi_{12}\left[^{\circ}\right]$ & $(-6 \pm 9)$ & {$[-37,13]$} \\
\hline$x$ & $(5.6 \pm 2.0) \cdot 10^{-3}$ & {$[1.4,9.6] \cdot 10^{-3}$} \\
$y$ & $(7.0 \pm 1.0) \cdot 10^{-3}$ & {$[5.0,9.1] \cdot 10^{-3}$} \\
$|q / p|-1$ & $(5.3 \pm 7.7) \cdot 10^{-2}$ & {$[-8.5,25.6] \cdot 10^{-2}$} \\
$\phi\left[^{\circ}\right]$ & $(-2.4 \pm 2.9)$ & {$[-8.8,3.7]$} \\
\hline$A_{\Gamma}$ & $(0.7 \pm 0.8) \cdot 10^{-3}$ & {$[-0.9,2.3] \cdot 10^{-3}$} \\
$A_{M}$ & $(11 \pm 14) \cdot 10^{-2}$ & {$[-15,44] \cdot 10^{-2}$} \\
$R_{M}$ & $(4.0 \pm 1.4) \cdot 10^{-5}$ & {$[1.7,7.2] \cdot 10^{-5}$} \\
$R_{D}$ & $(3.27 \pm 0.08) \cdot 10^{-3}$ & {$[3.10,3.44] \cdot 10^{-3}$} \\
$\delta_{K \pi}\left[^{\circ}\right]$ & $(18 \pm 12)$ & {$[-14,40]$} \\
$\delta_{K \pi \pi^{0}}\left[^{\circ}\right]$ & $(31 \pm 20)$ & {$[-11,73]$} \\
\hline$a_{\mathrm{CP}}^{\operatorname{dir}}\left(D^{0} \rightarrow K^{+} K^{-}\right)$ & $(-2.6 \pm 2.2) \cdot 10^{-3}$ & {$[-7.1,1.9] \cdot 10^{-3}$} \\
$a_{\mathrm{CP}}^{\operatorname{dir}}\left(D^{0} \rightarrow \pi^{+} \pi^{-}\right)$ & $(4.1 \pm 2.4) \cdot 10^{-3}$ & {$[-0.8,9.0] \cdot 10^{-3}$} \\
$\Delta a_{\mathrm{CP}}^{\mathrm{dir}}$ & $(6.6 \pm 1.6) \cdot 10^{-3}$ & {$[-9.8,3.5] \cdot 10^{-3}$} \\
\hline
\end{tabular}

Table 2. Results of the fit to $D$ mixing data. $\Delta a_{\mathrm{CP}}^{\mathrm{dir}}=a_{\mathrm{CP}}^{\mathrm{dir}}\left(D^{0} \rightarrow K^{+} K^{-}\right)-a_{\mathrm{CP}}^{\mathrm{dir}}\left(D^{0} \rightarrow \pi^{+} \pi^{-}\right)$.
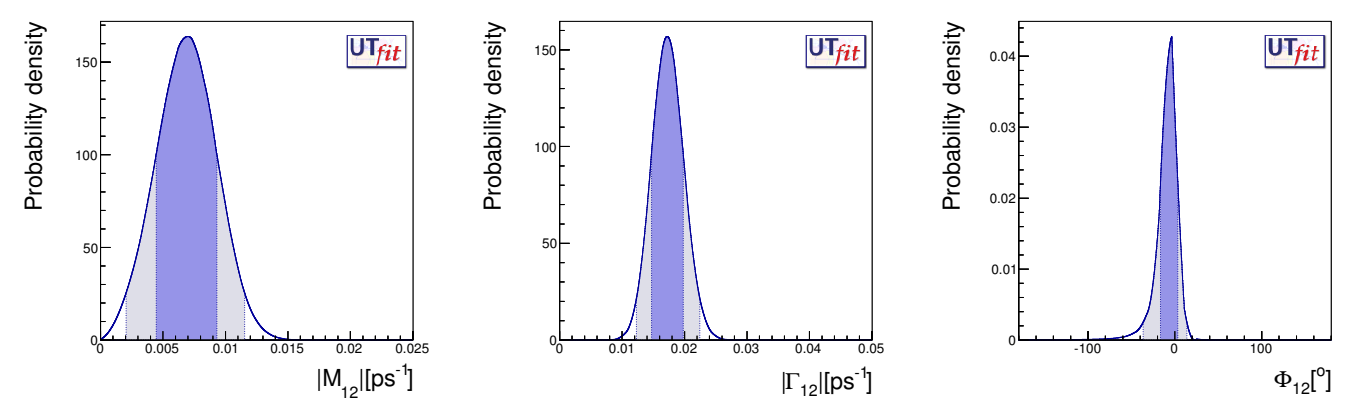

Figure 1. One-dimensional p.d.f. for the parameters $\left|M_{12}\right|,\left|\Gamma_{12}\right|$ and $\Phi_{12}$.

compatible results within errors. We notice, however, that HFAG performs a fit with four independent parameters $(x, y, \phi$ and $|q / p|)$, while in our analysis only three of these parameters are independent, as can be seen from eq. (3). With these assumptions, $\phi$ should vanish for $|q / p|=1$. This feature can be seen in figure 3 (up to the smoothing of the p.d.f) but not in the equivalent plot from HFAG, which displays completely different 2dimensional contours. We can but recommend that in the future HFAG takes the relation $\phi=\arg (y+i \delta x)-\arg \Gamma_{12}$ always into account.

The results in table 2 can be used to constrain NP contributions to $D-\bar{D}$ mixing and decays. 

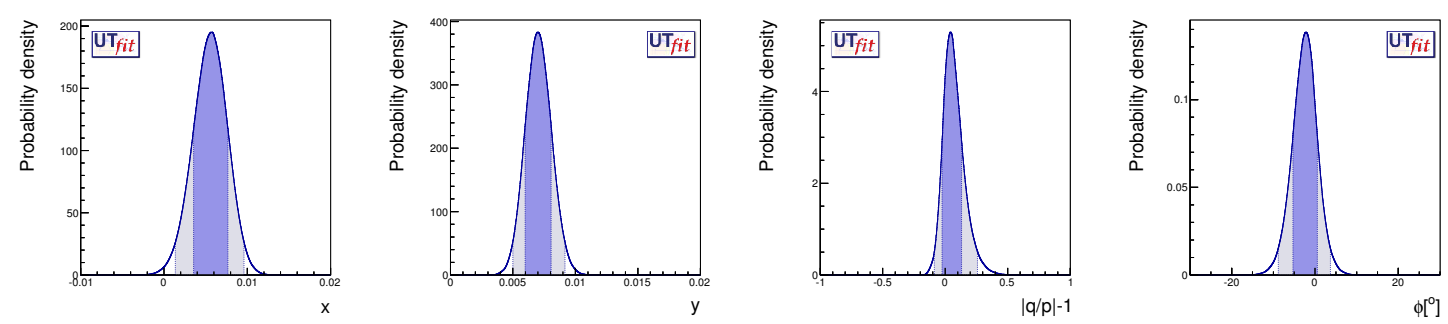

Figure 2. One-dimensional p.d.f. for the parameters $x, y,|q / p|-1$ and $\phi$.
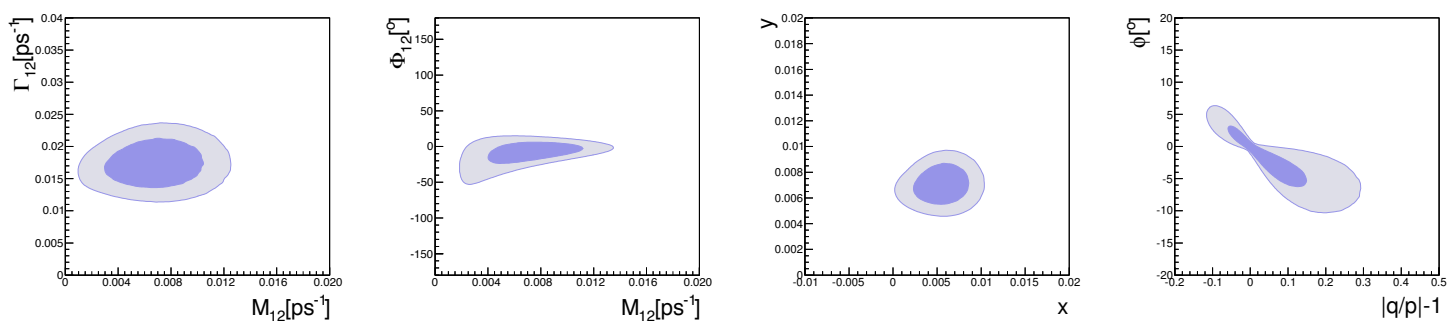

Figure 3. Two-dimensional p.d.f. for $\left|\Gamma_{12}\right|$ vs $\left|M_{12}\right|$ (top left), $\Phi_{12}$ vs $\left|M_{12}\right|$ (top right), $y$ vs $x$ (bottom left) and $\phi$ vs $|q / p|-1$ (bottom right).

\section{Acknowledgments}

M.C. is associated to the Dipartimento di Fisica, Università di Roma Tre. E.F. and L.S. are associated to the Dipartimento di Fisica, Università di Roma "La Sapienza". We acknowledge partial support from ERC Ideas Starting Grant n. 279972 "NPFlavour" and ERC Ideas Advanced Grant n. 267985 "DaMeSyFla". We thank B. Golob and A. Schwartz for clarifications about the HFAG averages.

Open Access. This article is distributed under the terms of the Creative Commons Attribution License which permits any use, distribution and reproduction in any medium, provided the original author(s) and source are credited.

\section{References}

[1] BaBAr collaboration, B. Aubert et al., Evidence for $D^{0}-\bar{D}^{0}$ mixing, Phys. Rev. Lett. 98 (2007) 211802 [hep-ex/0703020] [INSPIRE].

[2] Belle collaboration, M. Staric et al., Evidence for $D^{0}-\bar{D}^{0}$ mixing, Phys. Rev. Lett. 98 (2007) 211803 [hep-ex/0703036] [INSPIRE].

[3] BelLe collaboration, K. Abe et al., Measurement of $D^{0}-\bar{D}^{0}$ mixing parameters in $D^{0} \rightarrow K_{s}^{0} \pi^{+} \pi^{-}$decays, Phys. Rev. Lett. 99 (2007) 131803 [arXiv:0704.1000] [InSPIRE].

[4] M. Ciuchini et al., D- $\bar{D}$ mixing and new physics: general considerations and constraints on the MSSM, Phys. Lett. B 655 (2007) 162 [hep-ph/0703204] [INSPIRE].

[5] Y. Nir, Lessons from BaBar and Belle measurements of $D^{0}-\bar{D}^{0}$ mixing parameters, JHEP 05 (2007) 102 [hep-ph/0703235] [INSPIRE]. 
[6] E. Golowich, J. Hewett, S. Pakvasa and A.A. Petrov, Implications of $D^{0}-\bar{D}^{0}$ mixing for new physics, Phys. Rev. D 76 (2007) 095009 [arXiv:0705.3650] [InSPIRE].

[7] S. Fajfer, N. Kosnik and S. Prelovsek, Updated constraints on new physics in rare charm decays, Phys. Rev. D 76 (2007) 074010 [arXiv:0706.1133] [INSPIRE].

[8] UTfit collaboration, M. Bona et al., Model-independent constraints on $\Delta F=2$ operators and the scale of new physics, JHEP 03 (2008) 049 [arXiv:0707.0636] [INSPIRE].

[9] LHCB collaboration, R. Aaij et al., Evidence for CP-violation in time-integrated $D^{0} \rightarrow h^{-} h^{+}$ decay rates, Phys. Rev. Lett. 108 (2012) 111602 [arXiv:1112.0938] [INSPIRE].

[10] CDF collaboration, Improved measurement of the difference between time-integrated CP asymmetries in $D^{0} \rightarrow K^{+} K^{-}$and $D^{0} \rightarrow \pi^{+} \pi^{-}$decays at $C D F$, CDF public note 10784, http://www-cdf.fnal.gov/physics/new/bottom/120216.blessed-CPVcharm10fb/cdf10784.pdf, Fermilab, Batavia U.S.A. (2012).

[11] J. Brod, A.L. Kagan and J. Zupan, On the size of direct CP-violation in singly Cabibbo-suppressed D decays, Phys. Rev. D 86 (2012) 014023 [arXiv:1111.5000] [inSPIRE].

[12] D. Pirtskhalava and P. Uttayarat, CP violation and flavor $\mathrm{SU}(3)$ breaking in D-meson decays, Phys. Lett. B 712 (2012) 81 [arXiv:1112.5451] [InSPIRE].

[13] B. Bhattacharya, M. Gronau and J.L. Rosner, CP asymmetries in singly-Cabibbo-suppressed $D$ decays to two pseudoscalar mesons, Phys. Rev. D 85 (2012) 054014 [arXiv:1201.2351] [INSPIRE].

[14] H.-Y. Cheng and C.-W. Chiang, Direct CP-violation in two-body hadronic charmed meson decays, Phys. Rev. D 85 (2012) 034036 [Erratum ibid. D 85 (2012) 079903] [arXiv: 1201.0785] [INSPIRE].

[15] E. Franco, S. Mishima and L. Silvestrini, The Standard Model confronts CP-violation in $D^{0} \rightarrow \pi^{+} \pi^{-}$and $D^{0} \rightarrow K^{+} K^{-}$, JHEP 05 (2012) 140 [arXiv:1203.3131] [INSPIRE].

[16] J. Brod, Y. Grossman, A.L. Kagan and J. Zupan, A consistent picture for large penguins in $D \rightarrow \pi^{+} \pi^{-}, K^{+} K^{-}$, arXiv:1203.6659 [INSPIRE].

[17] FOCUS collaboration, J. Link et al., A measurement of lifetime differences in the neutral $D$ meson system, Phys. Lett. B 485 (2000) 62 [hep-ex/0004034] [INSPIRE].

[18] CLEO collaboration, S. Csorna et al., Lifetime differences, direct CP-violation and partial widths in $D^{0}$ meson decays to $K^{+} K^{-}$and $\pi^{+} \pi^{-}$, Phys. Rev. D 65 (2002) 092001 [hep-ex/0111024] [INSPIRE].

[19] Belle collaboration, K. Abe et al., A measurement of lifetime difference in $D^{0}$ meson decays, Phys. Rev. Lett. 88 (2002) 162001 [hep-ex/0111026] [INSPIRE].

[20] BaBAr collaboration, B. Aubert et al., Measurement of $D^{0}-\bar{D}^{0}$ mixing using the ratio of lifetimes for the decays $D^{0} \rightarrow K^{-} \pi^{+}, K^{-} K^{+}$and $\pi^{-} \pi^{+}$, Phys. Rev. D 78 (2008) 011105 [arXiv: 0712.2249] [INSPIRE].

[21] BABAR collaboration, B. Aubert et al., Measurement of $D^{0}-\bar{D}^{0}$ mixing using the ratio of lifetimes for the decays $D^{0} \rightarrow K^{-} \pi^{+}$and $K^{+} K^{-}$, Phys. Rev. D 80 (2009) 071103 [arXiv:0908.0761] [INSPIRE].

[22] Belle collaboration, A. Zupanc et al., Measurement of $y_{\mathrm{CP}}$ in $D^{0}$ meson decays to the $K_{S}^{0} K^{+} K^{-}$final state, Phys. Rev. D 80 (2009) 052006 [arXiv:0905.4185] [INSPIRE].

[23] LHCB collaboration, R. Aaij et al., Measurement of mixing and CP-violation parameters in two-body charm decays, JHEP 04 (2012) 129 [arXiv:1112.4698] [INSPIRE]. 
[24] Belle collaboration, M. Staric, New Belle results on D-D $\bar{D}$ mixing, talk presented at Charm 2012, Honolulu U.S.A. (2012).

[25] BABAR collaboration, N. Neri, HFAG mixing averages and other $e^{+} e^{-} B$ factory mixing results, talk presented at Charm 2012, Honolulu U.S.A. (2012).

[26] E791 collaboration, E. Aitala et al., Measurements of lifetimes and a limit on the lifetime difference in the neutral D meson system, Phys. Rev. Lett. 83 (1999) 32 [hep-ex/9903012] [INSPIRE].

[27] BaBAr collaboration, P. del Amo Sanchez et al., Measurement of $D^{0}-\bar{D}^{0}$ mixing parameters using $D^{0} \rightarrow K_{S}^{0} \pi^{+} \pi^{-}$and $D^{0} \rightarrow K_{S}^{0} K^{+} K^{-}$decays, Phys. Rev. Lett. 105 (2010) 081803 [arXiv: 1004.5053] [INSPIRE].

[28] E791 collaboration, E. Aitala et al., Search for $D^{0}-\bar{D}^{0}$ mixing in semileptonic decay modes, Phys. Rev. Lett. 77 (1996) 2384 [hep-ex/9606016] [INSPIRE].

[29] CLEO collaboration, C. Cawlfield et al., Limits on neutral D mixing in semileptonic decays, Phys. Rev. D 71 (2005) 077101 [hep-ex/0502012] [inSPIRE].

[30] BABAR collaboration, B. Aubert et al., Search for $D^{0}-\bar{D}^{0}$ mixing using semileptonic decay modes, Phys. Rev. D 70 (2004) 091102 [hep-ex/0408066] [InSPIRE].

[31] BABAR collaboration, B. Aubert et al., Search for $D^{0}-\bar{D}^{0}$ mixing using doubly flavor tagged semileptonic decay modes, Phys. Rev. D 76 (2007) 014018 [arXiv: 0705. 0704] [INSPIRE].

[32] Belle collaboration, U. Bitenc et al., Improved search for $D^{0}-\bar{D}^{0}$ mixing using semileptonic decays at Belle, Phys. Rev. D 77 (2008) 112003 [arXiv:0802.2952] [INSPIRE].

[33] BABAR collaboration, B. Aubert et al., Measurement of $D^{0}-\bar{D}^{0}$ mixing from a time-dependent amplitude analysis of $D^{0} \rightarrow K^{+} \pi^{-} \pi^{0}$ decays, Phys. Rev. Lett. 103 (2009) 211801 [arXiv: 0807 .4544] [INSPIRE].

[34] CLEO-C collaboration, W. Sun, D decay using quantum correlations, talk presented at Physics in Collision 2010, Karlsruhe Germany (2010).

[35] Belle collaboration, L. Zhang et al., Improved constraints on $D^{0}-\bar{D}^{0}$ mixing in $D^{0} \rightarrow K^{+} \pi^{-}$ decays at BELLE, Phys. Rev. Lett. 96 (2006) 151801 [hep-ex/0601029] [INSPIRE].

[36] BABAR collaboration, B. Aubert et al., Search for CP-violation in the decays $D^{0} \rightarrow K^{-} K^{+}$ and $D^{0} \rightarrow \pi^{-} \pi^{+}$, Phys. Rev. Lett. 100 (2008) 061803 [arXiv:0709.2715] [INSPIRE].

[37] Belle collaboration, M. Staric et al., Measurement of CP asymmetry in Cabibbo suppressed $D^{0}$ decays, Phys. Lett. B 670 (2008) 190 [arXiv:0807.0148] [INSPIRE].

[38] Heavy Flavor Averaging Group collaboration, D. Asner et al., Averages of b-hadron, c-hadron and $\tau$-lepton properties, arXiv:1010.1589 [INSPIRE].

[39] M. Ciuchini et al., 2000 CKM triangle analysis: a critical review with updated experimental inputs and theoretical parameters, JHEP 07 (2001) 013 [hep-ph/0012308] [INSPIRE].

[40] G.C. Branco, L. Lavoura and J.P. Silva, CP violation, Int. Ser. Monogr. Phys. 103 (1999) 1 [INSPIRE].

[41] G. Raz, Interpreting experimental bounds on $D^{0}-\bar{D}^{0}$ mixing in the presence of CP-violation, Phys. Rev. D 66 (2002) 057502 [hep-ph/0205113] [inSPIRE].

[42] A.L. Kagan and M.D. Sokoloff, On indirect $C P$-violation and implications for $D^{0}-\bar{D}^{0}$ and $B_{s}-\bar{B}_{s}$ mixing, Phys. Rev. D 80 (2009) 076008 [arXiv:0907.3917] [INSPIRE].

[43] Y. Grossman, Y. Nir and G. Perez, Testing new indirect CP-violation, Phys. Rev. Lett. 103 (2009) 071602 [arXiv:0904.0305] [INSPIRE]. 